\title{
Argumentos a fortiori
}

(A fortiori Arguments)

Hubert MARRAUD

Recibido: 29.04.2012

Versión final: 31.05.2012

BIBLID [0495-4548 (2014) 29: 79; pp. 99-112]

DOI: $10.1387 /$ theoria. 6275

RESUMEN: El objetivo de este artículo es describir los argumentos a fortiori en el marco de una teoría de los esquemas argumentativos de corte toulminiano. Una argumentación a fortiori comporta la comparación de la fuerza de dos o más argumentos, y requiere que esos argumentos involucren inferencias escalares. Se pueden distinguir dos órdenes de argumentos a fortiori, en función de si esa inferencia media entre las premisas y la conclusión o entre el respaldo y la garantía de los argumentos. El primer patrón corresponde al a fortior retórico y el segundo al a fortiori jurídico.

Palabras clave: a fortiori; analogía; esquema argumentativo; garantía; respaldo; topos.

ABSTRACT: I offer an account of a fortiori arguments in the context of a Toulmin-style theory of argumentation schemes. A fortiori arguments contain a comparison of the strength of two or more arguments involving scalar inferences. We can distinguish two kinds of a fortiori arguments depending on whether those scalar inferences connect the premises and the conclusion or the backing and the warrant of the arguments. The first pattern has been studied by rhetoricians and the second pattern by legal argumentation theorists.

Keywords: a fortiori; analogy; argumentation scheme; backing; warrant; topos.

Es ilógico, por supuesto, pero los que estudiamos filosofía inmoral nos regimos por algunas normas contradictorias.

(G. McDonald Fraser, Flashman el libertador)

\section{Introducción}

Los argumentos a fortiori no han recibido mucha atención en la teoría contemporánea de la argumentación. Si buscamos en Informal Logic y en Argumentation, dos de las revistas más representativas, encontramos un único artículo dedicado a este tipo de argumentos (Sartor 2005). Tampoco suelen aparecer en las clasificaciones de los esquemas argumentativos. En el compendio de Walton, Rees y Macagno (2007) se consignan sesenta esquemas argumentativos, que con las variantes de algunos de ellos ascienden a noventa y seis, pero entre ellos no está el argumento a fortiori. Quien se interese por los argumentos a fortiori tiene que acudir a la teoría de la argumentación jurídica y al ámbito francófono. Perelman y Plantin, por ejemplo, estudian el argumento a fortiori, y en

\footnotetext{
* Trabajo realizado en el marco del proyecto "La argumentación en la esfera pública: el paradigma de la deliberación" financiado por la Secretaría de Estado de Investigación, Desarrollo e Innovación (MEC), FFI2011-23125.
} 
teoría de la argumentación jurídica es tenido por uno de los argumentos interpretativos clásicos.

La suerte dispar del argumento a fortiori se explica por los conceptos de buen argumento predominantes en las distintas tradiciones. En el ámbito de habla inglesa, las teorías de la argumentación manejan fundamentalmente conceptos cualitativos de buen argumento, como el criterio RSA (relevancia-suficiencia-aceptabilidad) de la lógica informal. Según ese criterio, un buen argumento es aquél cuyas premisas son aceptables, relevantes y suficientes para la conclusión. No obstante este presupuesto ha sido cuestionado recientemente a partir de la reivindicación de Johnson (2000) de un nivel dialéctico en la evaluación de argumentos. Por el contrario el concepto de buen argumento dominante en el ámbito francófono, el de fuerza argumentativa, es comparativo. Puede comprobarse consultando el capítulo V de Perelman y Olbrechts-Tyteca (1989), en donde se dice que la de fuerza de los argumentos es una noción confusa pero indispensable, o Anscombre y Ducrot (1994), en donde se da la siguiente definición:

Un argumento A es más fuerte que un argumento B si y sólo si (1) en cualquier circunstancia y sea cual sea la conclusión C, si se usa B en favor de C, se debe considerar utilizable A para esa misma conclusión; (2) hay circunstancias en las que puede usarse A para una determinada conclusión C, sin considerar por ello que B es utilizable para C. (p. 51) ${ }^{1}$

$\mathrm{Si}$, como trataré de mostrar en este artículo, la argumentación a fortiori es una forma compleja de argumentación que comporta la comparación de dos o más argumentos, se entiende que su estudio esté ligado al desarrollo de un concepto comparativo de buen argumento.

\section{Antecedentes}

La denominación argumento a fortiori resulta tan problemática como la de argumento por analogía, si no más. Como es sabido J. S. Mill decía que "no hay ninguna palabra, sin embargo, que se use más laxamente, o en una mayor variedad de sentidos, que analogía" (1853, libro III, cap. XX, \3).

En su Diccionario de Filosofía Ferrater Mora distingue un sentido retórico y un sentido lógico de a fortiori. En su acepción retórica, un argumento a fortiori se caracteriza porque "contiene ciertos enunciados que se supone refuerzan la verdad de la proposición que se intenta demostrar" o porque "a una parte de lo que se aduce como prueba viene a agregarse la otra parte, sobreabundando en lo afirmado". Ferrater ofrece el ejemplo siguiente: Lope de Vega es un poeta, tanto más cuanto que en los pasajes de su obra en los que no pretendía expresarse poéticamente empleó un lenguaje predominantemente lírico. En su acepción lógica lo propio de los argumentos a fortiori es que sacan partido de la transitividad de adjetivos comparativos tales como "mayor que", "menor que", etc. Ferrater pone como ejemplo de argumento a fortiori en sentido lógico "Como Juan es más viejo que Pedro, y Pedro es más viejo que Antonio, Juan es más viejo que Antonio".

\footnotetext{
1 Aclaremos, para no fomentar malentendidos, que por argumento entienden Anscombre y Ducrot “razón”, y no la combinación de una razón y una tesis.
} 
Si nos atenemos a la descripción de Ferrater el término argumento a fortiori parece equívoco. En su acepción retórica el término remite a una forma de argumentación compleja, en la que se ofrecen dos o más razones para la misma conclusión. Eso emparenta a la argumentación a fortiori con formas de argumentación compleja como la argumentación múltiple y la argumentación coordinada. Por el contrario, los argumentos a fortiori en sentido lógico carecen de la complejidad que exhiben en su acepción retórica.

En la teoría de la argumentación jurídica encontramos la que parece una tercera acepción de argumento a fortiori. Aunque también aquí hay disparidad de opiniones, Tarello da una típica definición jurídica del argumento a fortiori: un argumento a fortiori es un procedimiento discursivo que "dada una norma jurídica que predica una obligación u otra calificación normativa de un sujeto o de una clase de sujetos, se debe concluir que valga (que sea válida, que exista) otra norma que predique la misma calificación normativa de otro sujeto o clase de sujetos que se encuentran en situación tal que merecen, con mayor razón que el primer sujeto o clase de sujetos, la calificación que la norma dada establece para el primer sujeto o clase de sujetos" (1980, 355).

Entendida al modo de Tarello, la argumentación a fortiori es una forma compleja de argumentación a partir de reglas. Según Schellens y De Jong $(2004,311)$ la argumentación a partir de reglas se usa para justificar la evaluación de una situación o proceso apelando a normas de evaluación o a normas de conducta (morales, legales o convencionales). Conforme a las definiciones al uso en la argumentación jurídica, una argumentación a fortiori involucra una norma y un supuesto de hecho diferente del previsto por el legislador para la aplicación de esa norma. Se pretende sin embargo que la norma es aplicable al nuevo supuesto "con mayor razón" que a los casos expresamente previstos por el legislador.

La comprensión jurídica del argumento a fortiori está más cerca de la acepción retórica de Ferrater que de la acepción lógica. Con todo, ambas se diferencian netamente. En primer lugar, en su acepción retórica un argumento a fortiori refuerza a otro argumento. Este es un rasgo enfatizado en el Diccionario Enciclopédico Hispanoamericano de 1887: el argumento a fortiori "Tiene por objeto refutar errores y precisar verdades ya reconocidas, fortaleciéndolas merced a determinadas comparaciones". Este carácter accesorio está sin embargo ausente de la acepción jurídica. En segundo lugar, en teoría de la argumentación jurídica el debate sobre los argumentos a fortiori se ha centrado en dos cuestiones principales: su relación con el argumento analógico y las formas del argumento a fortiori. Es notable a este respecto que ninguno de los ejemplos de argumentación a fortiori de Ferrater tiene nada que ver con la analogía, con independencia de cuál sea la manera de entenderla. Finalmente, tampoco los ejemplos usuales de argumentación jurídica a fortiori parecen corresponder a alguna de las acepciones de $\mathrm{Fe}$ rrater.

Así las cosas, parece haber tres acepciones mutuamente independientes de argumento a fortiori: retórica, lógica y jurídica. En el Compendio de Lógica, argumentación y retórica, entrada "A contrario/a fortiori/a pari/a simili, argumentos", Luis Vega recoge esas tres acepciones. Vega asocia la que venimos llamando acepción retórica con la locución "tanto más" y la que podemos llamar acepción jurídica con la locución "con mayor ra- 
zón”. Al mismo tiempo parece considerar más importantes esas dos acepciones que la acepción lógica, que considera como "un caso añadido", en el que los argumentos "envuelven una relación de transitividad en el contexto de una serie de comparaciones del tipo más_o mayor-que o menos-o menor-que".

\section{Argumentos por analogía}

Mi propósito es ofrecer un tratamiento unificado de los argumentos a fortiori. Siguiendo la opinión de Vega asumiré que el núcleo del concepto de argumento a fortiori lo forman las acepciones retórica y jurídica.

En teoría de la argumentación jurídica hay un cierto acuerdo en que los argumentos a fortiori están emparentados con los argumentos por analogía, ya se trate de una forma de argumentación autónoma, ya de una variante de los analógicos. Como la argumentación por analógía sí ha sido estudiada y debatida en la teoría de la argumentación contemporánea, su análisis puede ser un punto de partida adecuado para abordar el de los argumentos a fortiori.

En la tradición lógica (o quizá lógico-informal) los argumentos por analogía se entienden de dos maneras principales:

- "Por analogía" o "analógico" se refieren al vínculo entre las premisas y la conclusión, y por tanto se predican primariamente de inferencias. Zeleznikow y Hunter (1995) distinguen tres tipos de razonamiento: deductivo, inductivo y analógico, y Juthe (2005) distingue cuatro clases de argumentos: deductivos, inductivos, abductivos y analógicos.

- "Argumento por analogía" es el nombre de un esquema argumentativo; la denominación se sitúa pues en el mismo plano que argumento de autoridad, argumento de la parte al todo, etc. El argumento por analogía es uno de los 96 esquemas argumentativos del compendio de Walton, Reed y Macagno (2007). Asimismo este segundo enfoque es el habitual en teoría de la argumentación jurídica.

Cuando se entiende el argumento por analogía como un esquema argumentativo, hay dos descripciones básicas. Según la primera los argumentos por analogía se basan en la semejanza. Esta es la posición de los ya citados Walton, Reed y Macagno, quienes les atribuyen el siguiente patrón (Op.cit., 315s):

Premisa de semejanza: En general el caso C1 es similar al caso C2.

Premisa de base: $\quad$ A es verdadero (falso) en el caso C1.

Conclusión: $\quad$ A es verdadero (falso) en el caso C2.

También en la tipología pragmadialéctica de Garssen $(2007,31)$ el argumento por analogía es un especie de los argumentos por comparación del tipo semejanza.

La descripción alternativa ve los argumentos por analogía como metaargumentos. Es la posición de Woods y Hudak (1989) y Marraud (2007). En una argumentación por analogía intervienen dos o más argumentos, que desempeñan los papeles de foro y tema. Se alega entonces que las premisas del foro son a su conclusión lo que las pre- 
misas del tema son a la suya. Con esto se quiere decir que en ambos casos median las mismas relaciones lógicas. La analogía sirve entonces para transferir propiedades relativas a la relación entre las premisas y la conclusión del foro al tema. Eso supone considerar a los argumentos por analogía como argumentos acerca de argumentos.

Los argumentos por analogía son argumentos por paridad de razonamiento, por así decir. Son argumentos sobre argumentos, metaargumentos. Alegan que dos o más argumentos objeto valen o caen juntos, y que lo hacen porque están a la par en el aspecto pertinente $[. .$.$] Los argumentos objeto de los metaargumentos son así análogos$ entre sí. (Woods y Hudak, 1989, 127).

Un modo de atacar un argumento es preguntar si, en el contexto en el que ocurre, tiene la fuerza suficiente para establecer su conclusión. En una argumentación por analogía se pretende que el argumento cuestionado tiene la fuerza necesaria porque otro argumento, que no está en cuestión, la tiene.

\section{Dos análisis de una argumentación por analogía}

Mi tesis es que aunque hay argumentos por semejanza, muchas argumentaciones paradigmáticamente analógicas no pueden analizarse según ese patrón. Propongo por tanto distinguir esos dos esquemas argumentativos, reservando el nombre de argumento por analogía para un tipo de metaargumentos. El análisis de una argumentación "real", servirá para defender y precisar esta tesis.

"Del mismo modo que los americanos detienen a todos de los que sospechan que tienen lazos con Al Qaeda y los talibanes, aunque sea de forma remota, nosotros detuvimos a este hombre [Warren Weinstein] que lleva inmerso hasta el cuello en la ayuda americana a Pakistán desde los años 70", asegura Zawahiri en esa traducción [de la empresa de control de páginas web islamistas SITE Intelligence Group]. (D. Alandete, "Al Qaeda afirma que ha secuestrado a un estadounidense en Pakistán”. El País, 2/12/2011)

\section{Reconstrucción según el enfoque de semejanza}

P1: En general el proceder de los estadounidenses con los sospechosos de tener lazos con Al Qaeda y los talibanes es similar al de Al Qaeda con Warren Weinstein.

P2: El proceder de los estadounidenses está justificado / no está justificado.

C: El proceder de Al Qaeda con Weinstein está justificado/no está justificado.

Esta reconstrucción es pragmáticamente descabellada. Como es inverosímil que alZawahiri apruebe el proceder de los estadounidenses, hay que suponer que lo condena, pero en tal caso jel jefe de Al Qaeda también estaría condenando el secuestro de Weinstein por su propia organización! (que por cierto él califica significativamente de “detención”). Para salvar esta objeción se puede señalar que un aspecto pragmático importante para analizar el texto es que al Zawahiri se dirige a la opinión pública occidental, que presumiblemente considera legítimas las detenciones de yihadistas. Podría replicarse entonces que al Zawahiri usa un argumento en cuyas premisas no cree, que está adoptando el punto de vista de su oponente para persuadirle. Johnson mantiene que cuando el argumentador utiliza premisas que cree falsas, renuncia a la pretensión 
de persuadir racionalmente $(2000,338)$. Si Johnson está en lo cierto, la reconstrucción según el enfoque de semejanza comporta que el objetivo al Zawahiri no es la persuasión racional.

\section{Reconstrucción del enfoque metaargumentativo}

Desde un punto de vista metaargumentativo el jefe de Al Qaeda estaría diciendo que cualquier argumento que justifique el proceder de los estadounidenses con los sospechosos de tener lazos con Al Qaeda y los talibanes pude convertirse mutatis mutandis en un argumento que justifique el proceder de $\mathrm{Al}$ Qaeda con Weinstein. Eso no quiere decir que no pueda justificarse el proceder de Al Qaeda sin hacer lo propio con el de los EE.UU.- aduciendo, por ejemplo, que Al Qaeda estaría respondiendo a una agresión. De este modo, el mensaje de al Zawahiri es que Occidente no puede condenar, so pena de inconsistencia, el proceder de su organización con Weinstein. Se trataría, en suma, de un argumento ad hominem, en el sentido que esta expresión tenía en el siglo XVII. Esto es, su conclusión no es que una determinada conducta está justificada, sino que "esta persona está obligada a admitirlo en conformidad con sus principios de razonamiento, o por coherencia con su propia conducta, situación, etc." (R. Whately, Elements of Logic, 1838, 196). Este análisis de la argumentación de al Zawahiri me parece muy superior al análisis de semejanza, atendiendo a consideraciones pragmáticas, dialécticas y retóricas.

\section{Garantías y esquemas argumentativos}

Para describir las distintas formas de la argumentación a fortiori adaptaré los esquemas argumentativos al modelo de Toulmin, abandonando el modelo premisas-conclusión adoptado por Walton. La clasificación de los esquemas argumentativos se hace entonces según su garantía, es decir, del modo en que las premisas llevan a la conclusión. Esto es coherente con la idea de que los esquemas argumentativos son herramientas para la evaluación de argumentos, puesto que en el modelo de Toulmin la fuerza de un argumento depende precisamente de la garantía desplegada. "Las garantías son de diferente clase, por lo que confieren diversos grados de fuerza a las conclusiones que justifican" (Toulmin 2003, 137).

Para identificar el esquema ejemplificado por un argumento hay que darle el formato premisas por tanto conclusión porque garantía. Al formatearlo de ese modo no se pretende describir la forma del argumento. Como subraya Toulmin, la garantía no suele hacerse explícita, y su identificación forma parte de la evaluación del argumento, no de su reconstrucción. Sin premisas y conclusión no hay argumento, pero pueden ofrecerse unos enunciados para apoyar una conclusión sin tener una idea formada de cuál sea la justificación de la inferencia propuesta. Lo único que asume quien presenta un argumento es que la inferencia correspondiente está justificada. Es cierto que quien usa un argumento tiene la obligación de responder a la pregunta, si se le hace, ¿qué tienen que ver las premisas con la conclusión? Si no responde satisfactoriamente, habrá incumplido una obligación dialéctica, pero no habrá dejado de argumentar. La moraleja es que la 
garantía no es un componente de los argumentos en el mismo plano que las premisas o la conclusión.

Identificar el argumento de al Zawahiri como un argumento por analogía es asignarle el formato:

\begin{tabular}{|l|l|l|l|}
\hline $\begin{array}{l}\text { Los occidentales creen que hay } \\
\text { argumentos que justifican la } \\
\text { detención de los sospechosos de } \\
\text { tener lazos con Al Qaeda }\end{array}$ & $\begin{array}{l}\text { Por tanto } \\
\text { Porque }\end{array}$
\end{tabular}$\quad$\begin{tabular}{l}
$\begin{array}{l}\text { Los occidentales tienen que } \\
\text { admitir que hay argumentos } \\
\text { que justifican la detención de } \\
\text { Weinstein }\end{array}$ \\
\hline
\end{tabular}

Los campos argumentativos del tema y el foro son análogos

Los argumentos analógicos discurren acerca de relaciones argumentativas. Son por ello argumentos de segundo orden, metaargumentos. Se fundan en el principio de que los argumentos análogos tienen una fuerza similar. Su carácter metaargumentativo explica por qué muy a menudo los argumentos presentados como análogos son hipotéticos: el énfasis no está puesto en la aceptabilidad de las conclusiones, sino en la de las inferencias.

La fuerza de un argumento depende de su garantía y de su comparación con los argumentos concurrentes. De aquí se sigue, en primer lugar, que las garantías de los argumentos análogos tienen que ser similares y por tanto del mismo tipo. En teoría de la argumentación jurídica se dice que entre el foro y el tema debe apreciarse una identidad de razón. En segundo lugar, si la fuerza de un argumento se determina comparándolo con otros, para que dos argumentos tengan una fuerza parecida es preciso que para cada objeción o contraargumento que invalide uno de ellos, haya una objeción o contraargumento similar que invalide al otro. El campo de un argumento en una situación argumentativa está formado por todos los argumentos disponibles en esa situación que son pertinentes para su evaluación. Se puede concebir el campo de un argumento como un conjunto de argumentos parcialmente ordenado por la comparación de sus fuerzas. Dos argumentos son análogos si ocupan la misma posición dentro sus respectivos campos. De este modo la analogía entre argumentos remite a una analogía más general entre dos campos o dominios.

La ficha de un esquema argumentativo consta de una descripción de su forma y de una relación de cuestiones críticas que sirven como guía para evaluar los argumentos que se ajustan a ese patrón. Para terminar esta breve consideración de los argumentos por analogía se ofrece una relación indicativa de cuestiones críticas.

CC1. ¿Permiten la situación inicial y los objetivos del diálogo el uso de argumentos por analogía?

CC2. ¿La relación entre las premisas y la conclusión del foro es la requerida por la argumentación analógica?

CC3. ¿Cuál es la garantía invocada en el foro? ¿Es aplicable al tema?

CC4. ¿El respaldo aducido justifica debidamente la garantía del foro?

CC5. ¿Hay una correspondencia uno a uno entre los componentes del foro y del tema? 
CC6. ¿Están conectados en paralelo el foro y el tema?

CC7. ¿En qué medida se centra la analogía en relaciones?

CC8. ¿En qué medida es sistemática la analogía?

CC9. ¿Pueden acotarse los límites dentro de los que funciona la relación de analogía?

CC10. ¿Puede desarrollarse la analogía, dando lugar a correspondencias entre los campos del foro y el tema que inviertan la orientación argumentativa de la analogía?

Las cuestiones críticas pueden organizarse en cuatros grupos, que corresponden a otras tantas maneras de atacar un argumento. Un primer grupo lo forman preguntas relativas a las condiciones generales de uso del esquema (CC1). Las cuestiones del segundo grupo (CC2-CC3) inquieren por la justificación de las premisas y anticipan posibles objeciones, mientras que las del tercer grupo (CC6-CC9) tratan de identificar circunstancias excepcionales que impidan aplicar la garantía. Muchas veces las garantías son reglas que admiten excepciones y que solo autorizan el paso de las premisas a la conclusión en condiciones "normales". Cuando concurre una circunstancia excepcional, la garantía no es aplicable, la conclusión no puede inferirse de las premisas y el argumento que está siendo evaluado queda recusado. Finalmente, las cuestiones del último grupo (CC10) sirven para buscar posibles razones para negar la conclusión del argumento evaluado. Aun suponiendo que en una situación determinada un argumento proporcione una razón para aceptar su conclusión, tiene que ser sopesado con los argumentos en contrario disponibles para determinar si esa razón es suficiente. Las preguntas del cuarto grupo apuntan, por tanto, a posibles refutaciones del argumento evaluado.

\section{El a fortiori retórico como esquema argumentativo}

El origen de la noción de esquema argumentativo está en los tópicos de la dialéctica y la retórica antiguas. El concepto de argumento a fortiori puede remontarse al tópico aristotélico de lo más y lo menos: "Si al que más conviene el predicado no lo posee, es evidente que no lo poseerá aquél al que conviene menos" (Retórica II, 23, 1397b). Los ejemplos de Aristóteles incluyen tanto argumentos fácticos (si ni los dioses lo saben todo, menos aún los hombres) como normativos (si Héctor pudo matar con derecho a Patroclo, también a Aquiles Alejandro). Como no podía ser de otro modo, son a fortiori retóricos.

Los argumentos a fortiori se asemejan a los argumentos por analogía porque transfieren la fuerza de un argumento a otro. En las argumentaciones por analogía y a fortiori se aduce que un argumento es suficiente o insuficiente para mostrar que también lo es otro argumento. La diferencia es que en una argumentación por analogía se pretende que los dos argumentos tienen una fuerza similar y en una argumentación a fortiori que el segundo es (incluso) más fuerte que el primero.

Una primera variedad de argumentos a fortiori, que viene a corresponderse con su sentido retórico, compara la fuerza de dos argumentos cuya garantía es el mismo topos o correspondencia entre dos escalas. Anscombre y Ducrot $(1983,218)$ definen un topos 
como un principio general que sirve como garante del paso de las premisas a la conclusión y consiste en una correspondencia entre dos gradaciones no numéricas. Así el ejemplo aristotélico de los dioses y los hombres apela al topos $<+$ poderoso, + omnisciente $>$ (esta es una formulación provisional; cfr. $\int$.8). Es un topos directo, puesto que la correspondencia respeta el sentido de las escalas; en un topos inverso la correspondencia invierte el sentido al pasar de una escala a otra. Pero consideremos un ejemplo contemporáneo:

[...] estos primates [los chimpancés] son, sin lugar a dudas, las criaturas que más se asemejan a los seres humanos. Tienen conciencia de sí mismos, capacidad simbólica y cultura que transmiten de generación en generación; pueden aprender el lenguaje de los signos con un vocabulario de unas 300 palabras, y son incluso superiores a nosotros en algunas habilidades de memoria matemática. (J. de Jorge, "Una inteligencia casi humana", ABC, 15/08/2011)

Judith de Jorge alega que si la conciencia de sí mismos, la capacidad simbólica y cultural, y la capacidad de aprender el lenguaje de los signos son razones para atribuir inteligencia a los chimpancés (foro), su superioridad en algunas habilidades de memoria matemática constituye una razón aún más fuerte para esa misma conclusión (tema). Esto es, si la posesión de algunas capacidades intelectuales en menor grado que los humanos es una razón para considerar que los chimpancés son inteligentes, entonces la posesión de otras en un grado superior a los humanos es una razón más fuerte para esa misma conclusión. De Jorge compara el grado en el que chimpancés y humanos poseen una capacidad intelectual y correlaciona el resultado de esa comparación con la inteligencia para construir el topos <+(capacidad chimpancé/capacidad humano), + inteligencia $>$.

¿Qué es una razón más fuerte y cómo se determina? Como la fuerza de un argumento depende de su garantía, el incremento de la fuerza propio de los argumentos a fortiori debe explicarse en términos de una relación entre las garantías de los dos argumentos desplegados, el foro y en el tema. En los argumentos retóricos a fortiori la razón entre la fuerza del foro y del tema se explica porque esos argumentos comparten la misma garantía, que consiste en una correspondencia entre dos escalas no numéricas. Esas escalas no numéricas pueden no ser predicados graduales. En uno de los ejemplos de Aristóteles aparece el predicado "omnisciente", que no admite grados. En ese ejemplo, la garantía invocada es que a menos poder, menos esperable es la omnisciencia. Esto es, la graduación se refiere a la medida en que el primer factor constituye una razón para lo segundo, no a una hipotética cantidad de omnisciencia.

En suma, los argumentos retóricos a fortiori son metaargumentos cuya forma aproximada es

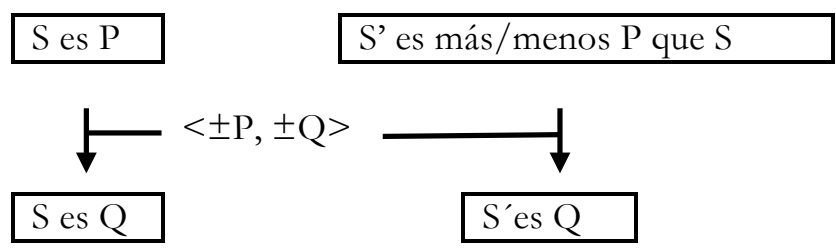

Aunque la cuestión requeriría una investigación histórica, me parece plausible que el a fortiori lógico surgiera del intento de dar un tratamiento deductivo de las inferencias escalares que están en la base del a fortiori retórico. 


\title{
7. El a fortiori jurídico como esquema argumentativo
}

Para explicar el funcionamiento de otras formas más complejas de argumentación $a$ fortiori hay que recurrir a la noción de respaldo ${ }^{2}$. Según Toulmin la función del respaldo es justificar la aplicabilidad general de la garantía $(2003,140)$. En los argumentos ad populum, por ejemplo, se alega que la conclusión es verdadera porque muchos la tienen por verdadera. Tradicionalmente se considera que los argumentos de ese tipo son falaces y que la garantía invocada no es válida. Cuando se cuestiona, no un argumento ad populum concreto, sino los argumentos ad populum en general, lo que se pide es que se respalde la garantía. Hahn y Oaksford $(2006,228)$ sugieren un respaldo para esta clase de argumentos: "Hay muchas pruebas de que la deliberación en grupo lleva a estimaciones y decisiones más precisas que la deliberación individual. [...] Esos efectos pueden explicarse por el hecho de que estadísticamente las muestras grandes tienden a reducir la varianza del error".

Resulta natural pensar que la relación entre el respaldo y la garantía se asemeja, al menos en algunos aspectos, a la relación entre las premisas y la conclusión (vid. Toulmin 2003, 144). Si es así, y la relación entre las premisas y la conclusión se analiza en términos de fuerza argumentativa, un respaldo puede proporcionar una justificación más o menos fuerte de la aplicabilidad de una garantía. Es decir, el puente para pasar del respaldo a la garantía podría consistir es un topos. Pues bien, los argumentos jurídicos a fortiori han de analizarse de esta manera.

Los argumentos jurídicos a fortiori comparan dos argumentos normativos. Lo característico de los argumentos normativos es que la garantía es una norma. Según la definición de Tarello, el argumento a fortiori concluye la existencia y aplicabilidad de una norma de la existencia de otra norma. Aunque esas normas son distintas, prescriben la misma calificación en dos situaciones distintas, aunque no con la misma fuerza. Tarello insiste en que el argumento a fortiori no se funda en la semejanza de los casos considerados, sino en la razón de la norma. Esa razón de la norma es justamente el respaldo de la garantía, que suele apelar a la voluntad del legislador.

Ezquiaga $(2008,144-146)$ analiza una argumentación a fortiori que toma de la sentencia 110/1984, de 26 de noviembre, del Tribunal constitucional español.

\begin{abstract}
El secreto profesional, es decir, el deber de secreto que se impone a determinadas personas, entre ellas los Abogados, de lo que conocieren por razón de su profesión, viene reconocido expresamente por la Constitución que en su artículo 24.2 dice que la Ley regulará los casos en que, por razón de parentesco o de secreto profesional, no se está obligado a declarar sobre hechos presuntamente delictivos. Evidentemente y a fortiori tampoco existe el deber de declarar a la Administración sobre esos hechos. La Constitución consagra aquí lo que es no un derecho sino un deber de ciertos profesionales que tiene una larga tradición legislativa (cfr. artículo 263 de la LECr).
\end{abstract}

Ezquiaga (Op.cit., p. 146) explica así el razonamiento que subyace al tema: "si ese artículo reconoce el secreto profesional frente a los jueces y tribunales, a fortiori lo reconoce frente a la Administración. ¿Por qué? Porque la Administración merece 'con mayor razón' que los jueces esa regulación".

\footnotetext{
${ }^{2}$ Lo mismo puede decirse de los argumentos por analogía.
} 
El párrafo $2^{\circ}$ del artículo 24.2 de la CE reza: "La ley regulará los casos en que, por razón de parentesco o de secreto profesional, no se estará obligado a declarar sobre hechos presuntamente delictivos." Como puede verse no especifica ante quién está permitido guardar silencio acerca de hechos presuntamente delictivos. Se interpreta no obstante que se refiere a la Administración de justicia porque el artículo 24 está dedicado a la protección judicial de los derechos y su punto segundo a los derechos del justiciable. Sin esta interpretación restrictiva no habría lugar al uso de un argumento $a$ fortiori.

El foro es un argumento a fortiori cuya garantía es la interpretación que hace el Tribunal Constitucional del art.24.2 de la Constitución Española, y que puede representarse así:

\begin{tabular}{|l|l|l|}
$\begin{array}{l}\text { Supongamos que una persona } \\
\text { sabe de un hecho presuntamente } \\
\text { delictivo por razón de su profesión }\end{array}$ & $\stackrel{\text { Por tanto }}{\longrightarrow}$ \\
Porque
\end{tabular}$\quad$\begin{tabular}{l}
$\begin{array}{l}\text { Esa persona no está obligada a } \\
\text { declarar sobre ese hecho ante la } \\
\text { Administración de justicia }\end{array}$ \\
\hline
\end{tabular}

El art. 24.2 de la Constitución reconoce el secreto

profesional ante la Administración de Justicia

Para construir el tema de la argumentación a fortiori se usa la misma premisa y se sustituye administración de justicia por administración en la conclusión. ¿Cuál es su garantía? Según la pauta usada, sería la norma resultante de hacer la misma sustitución en la garantía del foro. Ahora bien, en la interpretación del TC el derecho a no declarar no está amparado por el art.24.2 de la Constitución, sino por una norma no explícitamente promulgada. En su sentencia el TC intenta justificar la nueva norma (o, si se prefiere, la aplicación analógica de la antigua norma) aduciendo que en el art. 24.2 de la CE el legislador está consagrando "lo que es no un derecho sino un deber de ciertos profesionales". Lo que el TC está haciendo es, en términos toulmininianos, respaldar la garantía del tema. Para justificar la extensión de la norma se alega que si el legislador reconoce el secreto profesional ante el poder judicial, que es a quien compete establecer los hechos y que por ello debe tener mayores potestades que el ejecutivo para recabar de los ciudadanos las informaciones necesarias, con mayor razón lo reconocerá ante el poder ejecutivo, para quien por tanto supone una limitación menor. El paso del respaldo a la garantía se justifica invocando un topos inverso que correlaciona el reconocimiento de un derecho con los conflictos con otros fines deseables que ese reconocimiento pueda acarrear. Ese topos puede relacionarse fácilmente con la ley de ponderación de Alexy: "cuanto más alto sea el grado de incumplimiento o menoscabo de un principio, tanto mayor debe ser la importancia del otro" $(1988,147)$.

El respaldo apela a la voluntad del legislador, un lugar común en el tratamiento jurídico de los argumentos interpretativos. Claro que ese legislador es un legislador racional antes que el legislador real, y por tanto una ficción (Ezquiaga 1994, 91). La aplicación a fortiori del artículo 24.2 de la Constitución se justificaría porque el respaldo que hace que esa norma sea racionalmente aceptable, hace tanto más aceptable su versión ampliada. Así las cosas, la aceptación del artículo debería llevar al legislador a 
aceptar su extensión a los nuevos supuestos, so pena de irracionalidad. Esta argumentación es estructuralmente más compleja que la de Judith de Jorge, como evidencia su diagrama:

$$
\begin{array}{|l|}
\hline \text { Respaldo } 1 \\
\hline
\end{array}
$$

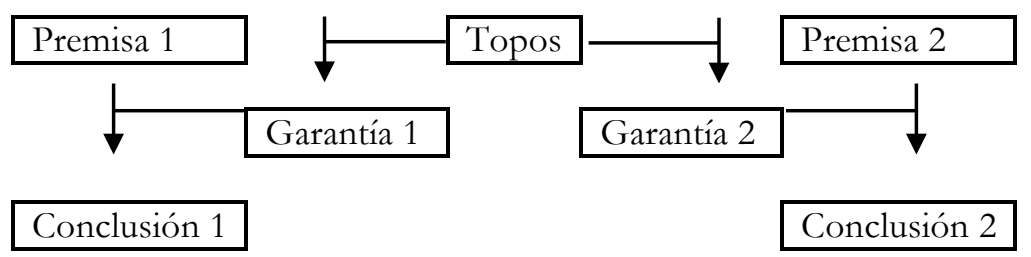

Una argumentación a fortiori invoca, lo mismo que una argumentación por analogía, una estructura común de relaciones argumentativas. El a fortiori jurídico involucra relaciones de orden superior a las del a fortiori retórico. En la argumentación de "Una inteligencia casi humana" la identidad de razón se sitúa en el paso de las premisas a la conclusión, y en la del Tribunal Constitucional en el paso del respaldo a la garantía. El modelo de Toulmin permite diferenciar estos dos órdenes de argumentos a fortiori. ${ }^{3}$

$\mathrm{El}$ argumento hipotético que concluye que quien tiene conocimiento de un hecho presuntamente delictivo por razón de su profesión no tiene obligación de declarar sobre ese hecho ante la Administración no es, pese a todo, más fuerte que el que concluye lo mismo con respecto a la Administración de justicia. No hay duda de que la ley establece ese derecho frente a jueces y magistrados, pero la intervención del TC no tendría sentido si esa certeza se extendiera a la Administración en general. Además, la Administración de justicia no deja de ser una parte de Administración. La mayor fuerza se refiere al apoyo que el reconocimiento del secreto profesional brinda a la interpretación ampliada del art.24.2 de la Constitución frente al que brinda a su interpretación restringida.

Situar la mayor razón en el tránsito del respaldo a la garantía permite dar cuenta de la debilidad relativa del argumento tema de una argumentación a fortiori. Esa debilidad se manifiesta en los presupuestos de su uso. El argumento a fortiori exige, como condición previa para su utilización, que no exista ninguna norma explícita aplicable a los supuestos del argumento tema. Si no se diera esa condición existiría un argumento normativo más fuerte que el argumento tema del a fortiori.

\section{Contraargumentos a fortiori}

El conector "ni siquiera" también puede ir asociado con una argumentación a fortiori. Una expresión canónica de argumentación a fortiori es "Si A es suficiente razón para B, tanto más lo es C para D". Cuando las conclusiones de los dos argumentos coinciden, pueden usarse en su lugar frases como "B porque A e incluso C" (como hace de Jor-

\footnotetext{
${ }^{3}$ En realidad introduce una jerarquía de argumentos a fortiori, puesto que podrían pedirse las credenciales del topos que media entre el respaldo y la garantía, y la respuesta podría ser un nuevo argumento que descansase en otro topos como garantía, y así indefinidamente.
} 
ge). Pero los argumentos a fortiori también tienen una forma negativa, en la que se trata de mostrar, no que un argumento tiene la fuerza requerida, sino que es demasiado débil. Frase como "Si A fuera suficiente razón para B, tanto más lo sería C para D” y "A no es suficiente razón para B, ni siquiera lo es C" expresan argumentaciones de ese tipo.

Uno de los ejemplos de Aristóteles es un contraargumento a fortiori: si ni los dioses lo saben todo, menos aún los hombres. El ejemplo compara la fuerza dos argumentos basados en el topos $<+$ poderoso, +esperablemente omnisciente $>$.

$$
\frac{\text { Los dioses son poderosos }}{\text { Los dioses lo saben todo }}>\frac{\text { Los hombre son poderosos }}{\text { Los hombres lo saben todo }}
$$

El argumento de la izquierda sería más fuerte que el de la derecha porque los dioses son más poderosos que los hombres. El argumentador pretende que el argumento de la izquierda es insuficiente, puesto que su premisa es verdadera y su conclusión falsa, e invita a inferir que entonces también lo es el de la derecha.

Para quien piense que el ejemplo de Aristóteles es demasiado artificial, he aquí una contraargumentación a fortiori tomada de la práctica.

[...] sólo una visión descarnada sobre la seguridad [...] podría sostener que la intervención estatal debería limitarse a eliminar el riesgo directo para las personas, al costo que fuere: pasándose por alto las garantías que apuntan a evitar o minimizar los castigos injustificados... Esta visión descarnada... no explica siquiera por qué la vida en sociedad debería ser defendida. Si se creyera, por ejemplo, como lo hace buena parte del pensamiento liberal, que sólo el consentimiento (real o hipotético) genera las condiciones de justificación para la existencia del Estado, sería difícil suponer que las personas accederían a vivir bajo una sociedad en la que ellas mismas podrían ser objetos potenciales del maltrato estatal. Es que, además, ni siquiera lo aconsejaría el cálculo 'racional' de costos y beneficios de una persona que descontara la posibilidad de ser ella misma objeto del aparato punitivo: si algo no es obvio es que el peligro que representa la delincuencia vaya a ser minimizado mediante el encierro y el maltrato de una persona. (González Bertomeu, J. y M.-P. Saffon, "Ni un preso más sin cupo", Sin Permiso, 06/12/10)

\section{Cuestiones críticas para los argumentos a fortiori}

La lista de cuestiones críticas para los argumentos a fortiori se asemeja a la de los argumentos por analogía, como era de esperar teniendo en cuenta la proximidad de los dos esquemas. Por ejemplo, con respecto a las condiciones generales de uso, los argumentos a fortiori y analógicos está tradicionalmente prohibido en el ámbito penal. Así lo establecen el art. 4.2 del Código Civil español y el artículo 14 de la Constitución mexicana, que menciona expresamente los argumentos a fortiori: "En los juicios del orden criminal queda prohibido imponer, por simple analogía, y aún por mayoría de razón, pena alguna que no esté decretada por una ley exactamente aplicable al delito de que se trata."

También serán similares las cuestiones acerca de posibles objeciones a las premisas, referidas ahora a la identificación del topos invocado y a si justifica y en qué medida el paso de las premisas a la conclusión del foro. Las cuestiones críticas orientadas a la recusación del argumento se refieren a si ese topos cubre igualmente el argumento tema, a la naturaleza de la correlación que establece y a los valores de su antecedente en las 
premisas de los argumentos comparados. Puede suceder, por ejemplo, que la correlación entre dos escalas sea directa hasta un umbral e inversa a partir de él.

Finalmente, y en relación a posibles refutaciones, ya se ha señalado que los argumentos jurídicos a fortiori son, lo mismo que los argumentos por analogía, más débiles que los argumentos que invocan una norma explícita.

\section{REFERENCIAS}

AA.VV. 1887. Diccionario Enciclopédico Hispano-Americano, "A Fortiori", tomo 1, 534-535. Barcelona: Montaner y Simón Editores.

Alexy, R. 1988. "Sistema jurídico, principios jurídicos y razón práctica". Doxa 5, 139-151.

Anscombre, J.-C. y Ducrot, O. 1994. La argumentación en la lengua. Madrid: Gredos.

Aristóteles 1998. Retórica. Madrid: Alianza.

Ezquiaga Ganuza, F.J. 1994. “Argumentos interpretativos y postulado del legislador racional”. Isonomía 1: 69-98.

-. 2008. La argumentación en la justicia constitucional. Medellín y Bogotá: Pontifica Universidad Javeriana.

Ferrater Mora, J. (1965). "A Fortiori” en Diccionario de Filosofia, 23. Buenos Aires: Editorial Sudamericana, $5^{\text {a }}$ edición.

Garssen, B. 2007. "Esquemas argumentativos" en R. Marafioti (ed.) Parlamentos, 19-36. Buenos Aires: Biblos.

Hahn, U. y Oaksford, M. 2006. "A Bayesian Approach to Informal Argument Fallacies". Synthese 152: 207-236.

Johnson, R.H. 2000. Manifest Rationality. Mahwah, NJ : Lawrence Erlbaum.

Juthe, A. 2005. "Arguments by Analogy", Argumentation 19: 1-27.

Marraud, H. 2007. "La analogía como transferencia argumentativa". Theoria 59: 167-188.

Mill, John Stuart. 1853. Sistema de lógica demostrativa e inductiva. Madrid: Rivadeneyra.

Perelman, C. 1980. La lógica jurídica y la nueva retórica. Madrid: Civitas.

—. y Olbechts-Tyteca, L. 1989. Tratado de la argumentación. La nueva retórica. Madrid: Gredos.

Sartor, G. 2005. "Reasoning with Factors". Argumentation 19: 417-432.

Schellens, Peter Jan y De Jong, Menno. 2004. "Argumentation Schemes in Persuasive Brochures”, Argumentation 18: 295-323.

Tarello, G. 1980. L’interpretazione della legge. Milán: Giuffrè Editore.

Toulmin, S.E. 2003. Los usos de la argumentación. Barcelona: Península.

Vega, L. y Olmos, P., eds. 2011. Compendio de lógica, argumentación y retórica. Madrid: Trotta.

Walton, D.N., Reed, C. y Macagno, F. 2008. Argumentation Schemes. Nueva York: Cambridge University Press.

Woods, J. y Hudak, B. 1989. "By Parity of Reasoning”, Informal Logic XI.3, 125-139.

Zeleznikow, J. y Hunter, D. 1995. "Deductive, inductive and analogical reasoning in legal decision support systems". Information \& Communications Technology Law 4, 2: 141-159.

Hubert Marraud es profesor titular de lógica y filosofía de la ciencia en la Universidad Autónoma de Madrid. Es autor de diversos artículos sobre teoría normativa de la argumentación y de los libros Methodus Argumentandi (2007) y ¿Es lógic@? (2013).

Dirección: Facultad de Filosofía y Letras, Universidad Autónoma de Madrid, Ciudad Universitaria de Cantoblanco, c/ Francisco Tomás y Valiente 1, 28049 Madrid, Spain. E-mail: hubert.marraud@uam.es 